\title{
Nouvelle plateforme en ligne "Guides de pratique Suisse»
}

\author{
Stefanie Hostettlera, Esther Kraft ${ }^{\mathrm{b}}$, Christoph Bosshard ${ }^{\mathrm{c}}$ \\ ${ }^{a} \mathrm{Dr}$ sc. EPFZ, division Données, démographie et qualité (DDQ) de la FMH \\ b lic. rer. oec., cheffe division Données, démographie et qualité (DDQ) de la FMH \\ c Dr méd., vice-président de la FMH, responsable du département Données, démographie et qualité (DDQ) de la FMH
}

Enoncés élaborés de façon systématique, les guides de pratique clinique (ou guidelines) doivent permettre de prendre des décisions fondées sur les preuves afin de fournir aux patients le meilleur traitement possible. Les guides de pratique sont nombreux et de nouveaux guides voient continuellement le jour, ce qui rend difficile toute vue d'ensemble et peut susciter des doutes quant à leur utilisation.

Forte de ce constat, l'Académie suisse pour la qualité en médecine (ASQM) de la FMH a démarré le projet de plateforme en ligne des guides de pratique Suisse [1] en décembre 2016 dans le but d'améliorer la documentation des guides de pratique et d'augmenter la transparence dans ce domaine.

\section{Objectifs du projet}

La plateforme en ligne vise avant tout à mettre à disposition des personnes intéressées des informations sur les guides de pratique clinique, à favoriser l'échange entre les utilisateurs et à les aider dans la rédaction de nouveaux guides. Dans le cadre de ce projet, il s'agira également de se pencher sur les questions suivantes:

- Approche critique de la qualité des guides

- Application uniforme des guides au sein d'une spécialisation médicale

- Echange interdisciplinaire et interprofessionnel

- Débat sur l'application des guides lors de polymorbidité

\section{Intérêt du projet}

La plateforme en ligne doit permettre de collecter des informations et de favoriser l'échange de connaissances et la transparence dans le domaine des guides de pratique clinique. Les guides documentés devront remplir des critères minimaux prédéfinis, p. ex. l'information sur leur financement devra être rendue publique, les liens d'intérêts des membres de la commission en charge du guide devront être documentés et la durée de validité du guide connue.

\section{Mise en œuvre du projet}

Dans le cadre de ce projet, la division Données, démographie et qualité (DDQ) de l'ASQM / la FMH a développé une structure de documentation et un questionnaire électronique en collaboration avec des experts. Les sociétés de discipline médicale, sociétés cantonales de médecine, hôpitaux/cliniques et autres organisations de la santé sont invités à documenter les guides de pratique qu'ils utilisent. L'enquête en ligne a démarré le 31 mai 2017 et dure jusqu'au 16 juillet 2017. La plateforme sera mise en ligne à fin août 2017. L'enquête sera reconduite une fois par année afin de compléter la plateforme et de l'actualiser.

\section{Participation à la plateforme en ligne}

En participant à ce projet, vous aidez à soutenir les guides de pratique de qualité et contribuez activement à améliorer la qualité des soins. La documentation ne prend que quelques minutes par guide. Les sociétés de discipline médicale, sociétés cantonales de médecine, hôpitaux/cliniques et autres organisations participant au projet seront mentionnés dans les différentes publications, sur les sites Internet, etc.

\footnotetext{
Référence

1 www.fmh.ch $\rightarrow$ ASQM $\rightarrow$ Projets relatifs à la qualité $\rightarrow$ Projets en cours $\rightarrow$ Plateforme en ligne "Guides de pratique Suisse»
} 